\title{
Molecular Characterization of Glycopeptide-Resistant Enterococci from Hospitals of the Picardy Region (France)
}

\author{
M. Biendo, ${ }^{1}$ C. Adjidé, ${ }^{2}$ S. Castelain, ${ }^{3}$ M. Belmekki, ${ }^{2}$ F. Rousseau, ${ }^{1}$ M. Slama, ${ }^{4}$ O. Ganry, ${ }^{2}$ \\ J. L. Schmit, ${ }^{5}$ and F. Eb ${ }^{1}$ \\ ${ }^{1}$ Service de Bactériologie, CHU Nord, Place Victor Pauchet, 80054 Amiens Cedex 1, France \\ ${ }^{2}$ Service d'Epidémiologie, Hygiène Hospitalière et Santé Publique, CHU Nord, Place Victor Pauchet, 80054 Amiens Cedex 1, France \\ ${ }^{3}$ Unité de Virologie clinique et fondamentale, Faculté de Médecine et de Pharmacie, 3 rue des Louvels, 80036 Amiens, Cedex, France \\ ${ }^{4}$ Service de Réanimation Néphrologique, CHU Sud, avenue René Laënnec, 80054 Amiens Cedex 1, France \\ ${ }^{5}$ Service de Pathologie Infectieuse, CHU Nord, Place Victor Pauchet, 80054 Amiens Cedex 1, France
}

Correspondence should be addressed to M. Biendo, biendo.maurice@chu-amiens.fr

Received 30 July 2010; Revised 15 September 2010; Accepted 17 September 2010

Academic Editor: William M. Shafer

Copyright ( 2010 M. Biendo et al. This is an open access article distributed under the Creative Commons Attribution License, which permits unrestricted use, distribution, and reproduction in any medium, provided the original work is properly cited.

We studied 138 glycopeptide-resistant enterococci (GRE) strains, consisting of 131 glycopeptide-resistant Enterococcus faecium (GREfm) and 7 glycopeptide-resistant Enterococcus faecalis (GREfs). The GREfm strains were resistant to penicillin, ampicillin, vancomycin, and teicoplanin, while the GREfs strains were only resistant to vancomycin and teicoplanin. The van $A$ gene was the only glycopeptide determinant present in all GRE isolates investigated. Genes coding for Hyl and Hyl+ Esp were detected in 39 $(29.8 \%)$ and $92(70.2 \%)$ of the 131 GREfm isolates, respectively. Three of the 7 GREfs were positive for gelE+asa 1 genes, 3 for gel E gene, and 1 for asa 1 gene. The genetic relationship between the 138 GRE was analyzed by pulsed-field gel electrophoresis (PFGE) and multilocus sequence typing (MLST). GREfm isolates were clustered in a single genogroup (pulsotype A), and GREfs were clustered in six genogroups (pulsotypes B-G). Among the isolates investigated by MLST, only 18 PCR products were sequenced (12 E. faecium and 6 E. faecalis), and 9 sequence types (STs) were identified.

\section{Introduction}

Enterococci form part of the normal flora of both the human and animal gastrointestinal tract but are also found in other anatomical sites including the vagina and oral cavity. Of the 20 enterococcal species known, Enterococcus faecalis and Enterococcus faecium are among the leading causes of several human infections, including bacteremia, septicemia, endocarditis, urinary tract infections, wound infections, neonatal sepsis, and meningitidis.

Glycopeptide-resistant enterococci (GRE) are a mutant of Enterococcus that originally developed in individuals exposed to antibiotics. They have increasingly emerged as a major cause of nosocomial infections worldwide [1]. This emergence has been associated with gradual replacement of Enterococcus faecalis by Enterococcus faecium and an epidemic rise of vancomycin-resistant E. faecium [2].
Vancomycin is the antibiotic of choice for infections caused by penicillin-resistant strains, alone or in combination with aminoglycosides. Acquired vancomycin resistance to this organism greatly reduces the number of treatment options and, therefore, constitutes a major therapeutic concern. This problem is further compounded by the fact that resistance genes can potentially be transferred to other pathogenic organisms such as Staphylococcus aureus.

GRE strains were reported for the first time in France and the United Kingdom in 1988 [3], and then in the USA [4]. In France, the incidence of glycopeptide resistance in E. faecium bacteremia is less than 5\% [3], the proportion of GRE is less than $2 \%$, and the prevalence rate has remained at $0.01 \%[5,6]$.

The main risk factor for the development of GRE strains is the excessive use of glycopeptides, but the use of third-generation cephalosporins and fluoroquinolones is also involved in the selection of GRE [7]. 
Three glycopeptide resistance phenotypes have been distinguished in the GRE strains on the basis of the level and inducibility of resistance to vancomycin and teicoplanin [8]. The Van A type is characterized by acquired inducible resistance to both vancomycin and teicoplanin [9]. Strains of the Van B type have acquired inducible resistance to various levels of vancomycin but not to teicoplanin [10]. Constitutive low-level resistance to vancomycin (Van C1, Van C2/3, Van $\mathrm{E}$, and Van $\mathrm{G}$ phenotypes) is an intrinsic property of motile enterococci, E. gallinarum, E. casseliflavus, and E. flavescens $[11,12]$. van $A$ and van $B$ are the main resistance genotypes reported for E. faecalis and E. faecium, the two species most frequently isolated from clinical sites. Numerous factors are associated with a greater risk of acquiring enterococcal infections. These factors, including antimicrobial resistance and expression of virulence factors associated with infectionderived E. faecalis and E. faecium strains, possess several putative virulence determinants, including aggregation substance, gelatinase, cytolysin, enterococcal surface protein, and hyaluronidase [13]. The first four virulence factors are found in E. faecalis, while the fourth and fifth virulence factors are specific for E. faecium [13].

Aggregation substance, encoded by asa1, which is carried on a plasmid, is a pheromone-inducible protein that enables the conjugative transfer of sex pheromone gene-containing plasmids through the clumping of one Enterococcus to another [14]. As a virulence factor, aggregation substance increases bacterial adherence to renal tubular cells [15] and heart endocardial cells [16].

Gelatinase, encoded by the chromosomal gelE, is an extra cellular zinc endopeptidase that hydrolyzes collagen, gelatine, and small peptides [17].

The production of cytolysin has also been shown to significantly worsen the severity of endocarditis [18]. Cytolysin genes are carried on a plasmid or are integrated into the bacterial chromosome [19]. Cytolysin consists of two components, lysine (L) and activator (A). The cytolysin operon consists of five genes, of which $c y l \mathrm{L1}, c y l \mathrm{L2}, \mathrm{cyl}$ $M$, and $c y l B$ are relevant to the expression of component $\mathrm{L}$, whereas $c y l A$ is necessary for the expression of component A.

The enterococcal surface protein, encoded by the chromosomal gene esp, has an interesting structure that includes a central core consisting of distinct tandem repeat units. Enterococcal surface protein is associated with increased virulence [20], colonization and persistence in the urinary tract, and biofilm formation [21].

Another virulence factor, hyaluronidase, was described in E. faecium [22]. The E. faecium hyaluronidase, encoded by the chromosomal gene $h y l$, shows homology to the hyaluronidases previously described in Streptococcus pyogenes, Staphylococcus aureus, and Streptococcus pneumoniae which are believed to contribute to invasion of the nasopharynx and pneumococcal pneumonia [23].

The aim of this study was to use pulsed-field gel electrophoresis (PFGE) and multilocus sequence typing (MLST) to characterize glycopeptide-resistant E. faecium (GREfm) and glycopeptide-resistant $E$. faecalis (GREfs) isolates from clinical specimens obtained from patients admitted to
Picardy hospitals (France). The van genotypes of the GRE isolates were determined, and the virulence factor genes were detected.

\section{Materials and Methods}

2.1. Setting. One hundred thirty-eight GRE clinical isolates obtained from 127 patients were collected from five hospitals in the Picardy region (Amiens University Hospital (AUH; 128 isolates), Picardy Private Hospital (PPH; 6 isolates), Montdidier hospital (MH; 2 isolates), Doullens Hospital (DH; 1 isolate), and Saint Quentin Hospital (SQH; 1 isolate)) between April 2004 and January 2009. Clinical isolates were recovered from rectal swabs $(n=103)$, from urine $(n=12)$, from pus $(n=6)$, from peritoneal fluid $(n=4)$, from blood $(n=3)$, from drainage tube $(n=2)$, from bile $(n=2)$, from urethral swab $(n=1)$, from vaginal swab $(n=1)$, from abscess $(n=1)$, from catheter $(n=1)$, from pyosalpinx $(n=1)$, and from sputum $(n=1)$ (Table 1$)$.

The medical records of all patients with GRE isolates were reviewed retrospectively. Clinical data collected included age, gender, the hospital and the ward in which these patients were hospitalized and where they came.

2.2. Defining Samples. In this study, the clinical samples are the samples taken for diagnosis, and the rectal swabs are the samples taken for screening.

2.3. Culture and Phenotypic Identification. Rectal swabs were cultured on Enterococcosel selective agar supplemented with $8 \mu \mathrm{g} / \mathrm{mL}$ vancomycin (Becton Dickinson, France) with teicoplanin disc. Clinical samples were cultured on Columbia agar supplemented with 5\% defibrinated horse blood, and both were incubated aerobically for $24-48$ hours at $37^{\circ} \mathrm{C}$. Isolates were identified as E. faecium or E. faecalis by rapid ID32 Strep system according to the manufacturer's instructions (BioMérieux, France).

2.4. Antimicrobial Susceptibility Testing. Resistance to vancomycin, teicoplanin, penicillin, and ampicillin and to kanamycin, gentamicin, and streptomycin was tested by disc diffusion methods according to the Comite de l'Antibiogramme de la Société Française de Microbiologie (CASFM) guidelines [24]. Minimum inhibitory concentrations (MICs) of these antimicrobial agents were determined using E-test strips (BioMérieux, France), and the results were interpreted according to established breakpoint values [24]. E. faecium Van A CIP 104676 and E. faecalis Van B CIP 104105 standard strains were used as reference strains.

\subsection{Identification of GRE and Glycopeptide Resistance Deter-} minants. Total DNA was extracted from enterococci by using the BioRobot EZ1 extractor apparatus (QIAGEN, France) according to the manufacturer's recommendations. In order to determine the genotype responsible for glycopeptide resistant strains, we used a multiplex PCR (MPCR) as previously described [25]. During the mPCR, the DNA fragments were identified according to their size. This MPCR allowed 
TABLE 1: Frequency of glycopeptide-resistant enterococci (GRE) in relation to the number of clinical samples.

\begin{tabular}{lccc}
\hline Source & Specimen & Absolute number of GRE isolates & Relative frequency $(\%)$ \\
\hline Hospital surveillance & Rectal swabs & 103 & 74.7 \\
& Urine & 12 & 8.8 \\
& Pus & 6 & 4.4 \\
& Peritoneal fluid & 4 & 2.9 \\
& Blood & 3 & 2.2 \\
& Drainage tube & 2 & 1.4 \\
Hospital clinical samples $(n=35)$ & Bile & 2 & 1.4 \\
& Urethral swab & 1 & 0.7 \\
& Vaginal swab & 1 & 0.7 \\
& Abscess & 1 & 0.7 \\
& Catheter & 1 & 0.7 \\
& Pyosalpinx & 1 & 0.7 \\
\hline
\end{tabular}

TABLE 2: Oligonucleotide primers used to amplify van genes.

\begin{tabular}{lccc}
\hline Amplified gene & Oligonucleotide sequence $\left(5^{\prime} \rightarrow 3^{\prime}\right)$ & Position & PCR product size $($ bp $)$ \\
\hline \multirow{2}{*}{ van $A$} & $\mathrm{~A}_{1}-5^{\prime}$-GGG-AAA-ACG-ACA-ATT-GC-3' & $175-191$ & 732 \\
& $\mathrm{~A}_{2}-5^{\prime}$-GTA-CAA-TGC-GGC-CGT-TA-3' & $907-891$ & $173-189$ \\
van $B$ & $\mathrm{~B}_{1}-5^{\prime}$-ATG-GGA-AGC-CGA-TAG-TC-3' & $807-791$ & 635 \\
& $\mathrm{~B}_{2}-5^{\prime}$-GAT-TTC-GTT-CTT-CGA-CC-3' & $246-272$ & 822 \\
van $C-1$ & $\mathrm{C}_{1}-5^{\prime}$-GGT-ATC-AAG-GAA-ACC-TC-3' & $1067-1051$ & $455-486$ \\
van $C-2$, van $C-3$ & $\mathrm{C}_{2}-5^{\prime}$-CTT-CCG-CCA-TCA-TAG-CT-3' & $885-869$ & 439 \\
\hline
\end{tabular}

the simultaneous detection of glycopeptide resistance genotypes: van $A$, van $B$, van $C 1$, and van $C 2 / 3$ (Table 2). PCR was performed on a DNA thermal cycler (model MJ, MINI Gradient, BioRad, France) in a final volume of $50 \mu \mathrm{L}$ containing $25 \mu \mathrm{L}$ GoTaq Green Master Mix (Promega, USA), 20 pmol of each oligonucleotide primer pair, and $1 \mu \mathrm{L}$ of DNA as template. The cycling conditions were $94^{\circ} \mathrm{C}$ for 2 minutes followed by 30 cycles of $94^{\circ} \mathrm{C}$ for 1 minute, $54^{\circ} \mathrm{C}$ for 1 minute, $72^{\circ} \mathrm{C}$ for 1 minute, and then $72^{\circ} \mathrm{C}$ for 10 minutes. PCR products were resolved by electrophoresis on a $1 \%$ agarose-Tris-EDTA gel containing $0.5 \mu \mathrm{g}$ per $\mathrm{mL}$ of ethidium bromide. Smartladder (Eurogentec, Belgium) was used as molecular weight marker.

After the PCR test, the PCR products obtained were hybridized with membrane strips coated with E. faecium, E. faecalis, E. gallinarum, and E. casseliflavus species using the specific probes provided with the Genotype Enterococcus kit (Hain Lifescience GmbH, Germany). The hybridization procedures were performed according to the manufacturer's recommendations. This technique was used to confirm combined identification of enterococcal species and van resistance genes.

2.6. Detection of Genes Encoding GRE Virulence Factors by Multiplex PCR. The presence of five genes encoding virulence factors in GRE isolates [14, 22, 26] were investigated by multiplex PCR using the oligonucleotide primer pairs previously reported [10] (Table 3), for which primers were designed based on published DNA sequences from the GenBank database. The choice of these five genes was based, for which they constitute the principal virulence factor genes reported for E. faecalis and E. faecium strains, on the high frequency reported in Europe [3] and in France [27] in enterococci strains, and on their use in mPCR schemes [13]. PCR was performed as described above. Each $50 \mu \mathrm{L}$ PCR mixture consisted of $25 \mu \mathrm{L}$ Gotaq Green Master Mix, 20 pmol of each oligonucleotide primer pair for asal, gel E, hyl, cyl $A$, and esp, and $5 \mu \mathrm{L}$ of DNA as template. Amplifications were performed under the following conditions: $95^{\circ} \mathrm{C}$ for 15 minutes, followed by 30 cycles of 1 minute at $94^{\circ} \mathrm{C}, 1$ minute at $56^{\circ} \mathrm{C}, 1$ minute at $72^{\circ} \mathrm{C}$, and then 10 minutes at $72^{\circ} \mathrm{C}$ for the last cycle. PCR products were then sequenced.

2.7. PFGE. Macrorestriction analysis by PFGE was performed for $131 \mathrm{E}$. faecium and $7 \mathrm{E}$. faecalis isolates with SmaI-restricted whole-cell DNA embedded in 1\% agarose plugs and separated in a $1.2 \%$ pulsed-certified agarose gel with a contour-clamped Homogeneous Electric Field (CHEF DRII apparatus; BioRad, France). E. faecium Van A CIP 104676 and E. faecalis Van B CIP 104105 strains were used as reference strains. Concatemers of bacteriophage $\lambda$-ladder were used as molecular weight marker (BioRad, France). 
TABLE 3: Oligonucleotide primers used to amplify virulence factor genes.

\begin{tabular}{|c|c|c|c|c|}
\hline Gene & Virulence factor & Primer name & Oligonucleotide sequence $\left(5^{\prime}\right.$ to $\left.3^{\prime}\right)$ & $\begin{array}{l}\text { Product } \\
\text { size (bp) }\end{array}$ \\
\hline \multirow{2}{*}{ asal } & \multirow{2}{*}{$\begin{array}{l}\text { Aggregation substance, encoded by the } \\
\text { plasmid asa1 }\end{array}$} & ASA 11 & GCA-CGC-TAT-TAC- GAA -CTA-TGA & \multirow{2}{*}{375} \\
\hline & & ASA 12 & TAA-GAA-AGA-ACA-TCA-CCA-CGA & \\
\hline \multirow{2}{*}{ gelE } & \multirow{2}{*}{$\begin{array}{l}\text { Gelatinase, encoded by the chromosomal } \\
\text { gelE }\end{array}$} & GEL 11 & TAT-GAC-AAT-GCT-TTT-TGG-GAT & \multirow{2}{*}{213} \\
\hline & & GEL 12 & AGA-TGC-ACC-CGA-AAT-AAT-ATA & \\
\hline \multirow{2}{*}{ cylA } & \multirow{2}{*}{ Cytolysin, encoded by the plasmid cylA } & CYT I & ACT-CGG-GGA-TTG-ATA-GGC & \multirow{2}{*}{688} \\
\hline & & CYT II & GCT-GCT-AAA-GCT-GCG-CTT & \\
\hline \multirow{2}{*}{ esp } & \multirow{2}{*}{$\begin{array}{l}\text { Enterococcal surface protein, encoded by } \\
\text { the chromosomal esp }\end{array}$} & ESP $14 \mathrm{~F}$ & AGA-TTT-CAT-CTT-TGA-TTC-TTG-G & \multirow{2}{*}{510} \\
\hline & & ESP $12 R$ & AAT-TGA-TTC-TTT-AGC-ATC-TGG & \\
\hline \multirow{2}{*}{$h y l$} & \multirow{2}{*}{$\begin{array}{l}\text { Hyaluronidase, encoded by the } \\
\text { chromosomal hyl }\end{array}$} & HYL n1 & ACA-GAA-GAG-CTG-CAG-GAA-ATG & \multirow{2}{*}{276} \\
\hline & & HYL n2 & GAC-TGA-CGT-CCA-AGT-TTC-CAA & \\
\hline
\end{tabular}

TABLE 4: Amplification and sequencing primers for $d d l, g y d, g d h$, and psts.

\begin{tabular}{lccc}
\hline Gene & Primer name & Oligonucleotide sequence $\left(5^{\prime}\right.$ to $\left.3^{\prime}\right)$ & Product size $($ bp $)$ \\
\hline \multirow{2}{*}{$d d l$} & DDL1 & GAG-ACA-TTG-AAT-ATG-CCT-TAT-G & 465 \\
& DDL2 & AAA-AAG-AAA-TCG-CAC-CG & \\
GDH1 & GGd & GDC-GCA-CTA-AAA-GAT-ATG-GT & 530 \\
& GY2 & CCA-AGA-TTG-GGC-AAC-TTC-GTC-CCA & 556 \\
psts & GYD2 & CAA-ACT-GCT-TAG-CTC-CAA-GGC & 583 \\
& PSTS1 & CAT-TTC-GTT-GTC-ATA-CCA-AGC & \\
\hline
\end{tabular}

$d d l$ : D-alanine-D-alanine ligase, $g d h$ : glucose-6-phosphate dehydrogenase, gyd: glyceraldehyde-3-phosphate dehydrogenase, and psts: phosphate ATP-binding cassette transporter.

PFGE patterns were interpreted according to the criteria of Tenover et al. [28]. The similarity dendrogram was constructed with the unweighted pair-group method with arithmetic means (UPGMA) using the DICE correlation coefficient.

2.8. MLST Sequence Types. Four housekeeping genes (loci) (Table 4) were amplified for each isolate [29]. The choice of these housekeeping genes was based on their putative function and on their use in MLST schemes of E. faecium. Information on these loci is available at the MLST web site (http://efaecium-mslt.net).

2.9. PCR. Internal 400- to 600-bp gene fragments were amplified by PCR. Reactions were performed in $50 \mu \mathrm{L}$ volumes composed of $25 \mu \mathrm{L}$ GoTaq Green Master Mix, 20 pmol of each oligonucleotide primer pair, and $1 \mu \mathrm{L}$ of bacterial DNA as template. PCR conditions for all amplification reactions were as follows: $94^{\circ} \mathrm{C}$ for 3 minutes, 35 cycles at $94^{\circ} \mathrm{C}$ for 30 seconds, $50^{\circ} \mathrm{C}$ for 30 seconds, $72^{\circ} \mathrm{C}$ for 30 seconds, and $72^{\circ} \mathrm{C}$ for 5 minutes The amplicons were analyzed by electrophoresis as described above.

2.10. MLST Data Analysis. Sequences of each allele were trimmed and compared with all alleles in the MLST database. Each unique nucleotide sequence was assigned a unique allele number. The allele profile for each isolate was determined and consisted of a line listing of allele numbers for each gene. Isolates were then assigned a sequence type (ST) according to their allelic profiles.

2.11. Nucleotide Sequence Accession Numbers. The sequences of van genes, virulence factor genes, and the alleles housekeeping genes have been given the following GenBank accession numbers: HM641733 (van A), HM 641734 (asa1), HM 641735 (gel E), HM 641736 (psts), HM 641737 (gdh), and HM 641738 (gyd) for E. faecalis; HM 641739 (van A), HM 641740 (hyl), HM 641741 (esp), HM 641742 (psts), HM 641743 ( $g d h)$, HM 641744 ( $g y d$ ), and HM 641745 (esp) for E. faecium.

\section{Results}

3.1. Epidemiology. GRE was isolated from a total of 127 patients hospitalized during the period from April 2004 through January 2009. The first GRE isolates observed were two E. faecalis strains isolated from urinary tract infection of patients hospitalized in the AUH nephrology and orthopedic wards (April and May 2004, resp.). From 2005 to 2008, five other GREfs were isolated (three from rectal swabs and two from vaginal swab and pus) from patients hospitalized in AUH wards. 
From July to November 2005, during an outbreak comprising a limited number of cases, 33 GREfm (23 from rectal swabs and 10 from peritoneal fluid, blood, drainage tube, pus, bile, and catheter) were obtained from 25 colonized/infected patients, also hospitalized in AUH wards.

The major outbreak occurred from May 2006 to January 2009. Ninety-eight GREfm (22 isolates from clinical samples and 76 isolates from screening rectal swabs) were obtained from 95 patients hospitalized at AUH (85 patients), $\mathrm{PPH}$ (6 patients), MH (2 patients), DH (1 patient), and SQH (1 patient). The most recent isolates were obtained during fecal screening of all hospitalized contact patients, as part of infection control measures.

3.2. Patients Carrying GRE Isolates. Of 127 hospitalized patients included in this study, 67 (52.7\%) were men and $60(47.3 \%)$ were women. The mean age of these patients was 70.1 years (range: 19-98 years) in men and 73.6 years (range: $16-95$ years) in women (M/F sex ratio: 1 : 12). These patients were classified as either colonized, $74.8 \%$ (95/127), or infected, $25.2 \%$ (32/127), according to the definitions of French guidelines [30] based on those of the Centers for Disease Control and Prevention [31]. This distribution confirms the low ratio of infections versus colonization. The patient distribution according to GREpositive specimens showed that 94 patients had one GREpositive surveillance rectal swab and 1 patient had 3 GREpositive surveillance rectal swabs; twenty-four patients had one GRE-positive clinical sample, and 8 patients had 11 GRE-positive clinical samples plus 6 GRE-positive surveillance rectal swabs. Application of infection control measures included weekly surveillance cultures and environmental decontamination guided by culture and PCR-hybridization results.

3.3. PCR Assays and Sequencing Results. Sequencing yielded five distinct DNA sequences: one from Van A PCR (732 bp), one from Esp (510bp), one from Asa 1 (375bp), one from Hyl (276 bp), and one from Gel E (213bp). The nucleotide and amino acid sequences of van gene and virulence factor genes obtained were compared to the known sequences of Genbank. The nucleotide and amino acid sequences of the van $A$ gene had $100 \%$ genetic identity and $100 \%$ amino acid identity with E. faecium pIP816 plasmid, accession $\mathrm{n}^{\circ}$ AM932524. The esp gene sequences exhibited a $100 \%$ nucleotide and amino acid homology with sequences for E. faecium isolate E470 putative enterococcal surface protein (esp) gene, accession $n^{\circ}$ AY322500. The nucleotide and amino acid sequences of asal gene from our $E$. faecalis strains were identical to those known of $E$. faecalis plasmid pAD1 asal gene for aggregation substance (100\% genetic and amino acid identity), accession $n^{\circ} \mathrm{X} 17214$. The Hyl sequences showed best similarity with $E$. faecium putative hyaluronidase $(h y l)$ gene (100\% genetic identity; $100 \%$ amino acid identity), accession $\mathrm{n}^{\circ} \mathrm{AF} 544400$, while the partial Gel $E$ sequences showed best similarity with $E$. faecalis gelatinase ( $\mathrm{gel} E)$ gene (100\% genetic identity; $100 \%$ amino acid identity), accession $\mathrm{n}^{\circ} \mathrm{M} 37185$.
3.4. Molecular Identification, Antibiotic Susceptibility, and Virulence Factors. The molecular identification of 138 GRE showed that 131 enterococci isolates belonged to the $E$. faecium species $(94.9 \%)$ and 7 (5.1\%) belonged to the E. faecalis species. The van $A$ gene was the only glycopeptide resistance determinant found in all isolates studied. The resistance patterns for the isolates are shown in Table 5. The 131 GREfm isolates were resistant to penicillin (MICs, 96 to $>256 \mu \mathrm{g} / \mathrm{mL}$ ) and ampicillin (MICs, 48 to $>256 \mu \mathrm{g} / \mathrm{mL}$ ). One hundred twenty-eight of these isolates showed HLKR [(high-level kanamycin resistance) (MICs, $>256$ to $>512 \mu \mathrm{g} / \mathrm{mL}$ )], 116 showed HLGR [(high-level gentamicin resistance) (MICs, $>256$ to $>512 \mu \mathrm{g} / \mathrm{mL}$ )], and 53 showed HLSR [(high-level streptomycin resistance) (MICs, $>256$ to $>512 \mu \mathrm{g} / \mathrm{mL}$ )]. The 7 GREfs were susceptible to penicillin (MICs, 1.5$4 \mu \mathrm{g} / \mathrm{mL}$ ) and ampicillin (MICs, $0.50-1.5 \mu \mathrm{g} / \mathrm{mL}$ ). Six isolates showed HLKR (MICs of $>512 \mu \mathrm{g} / \mathrm{mL}$ ) and HLGR (MICs, $>256$ to $>512 \mu \mathrm{g} / \mathrm{mL}$ ), and one isolate showed HLSR (MIC, $>512 \mu \mathrm{g} / \mathrm{mL}$ ). Glycopeptide susceptibility test results were in agreement with resistance genotypes: the MICs of vancomycin were $>256 \mu \mathrm{g} / \mathrm{mL}$, and the MICs of teicoplanin were 32 to $>256 \mu \mathrm{g} / \mathrm{mL}$. Genes coding for Hyl and Hyl+ Esp were detected in $39(29.8 \%)$ and $92(70.2 \%)$ of the 131 GREfm isolates, respectively. Three of the 7 GREfs were positive for gel E+ asa 1 genes, 3 were positive for gel $E$ gene, and 1 was positive for asa 1 gene (Table 5). The cyl A gene was not detected in any of the GRE isolates examined.

3.5. Molecular Typing and Clonal Characteristics of GRE. Analysis of PFGE profiles showed that 131 GREfm isolates shared a similar electrophoretic profile, designated type A, and were clonally related. This PFGE type A encompassed 26 different subtypes (A1-A26). Subtypes A16 and A26 each corresponded to two isolates. The other subtypes corresponded to single isolate. The seven GREfs isolates appeared to be more heterogeneous on the basis of their PFGE profiles in six different types (B-G). Only type $G$ was identified in two isolates with identical profiles. These two isolates were obtained from two different patients hospitalized in different wards. These pulsotypes were considered to be sporadic profiles [32].

3.6. Diversity of Housekeeping Genes. MLST PCR was performed for all 138 isolates belonging to PFGE types AG, but only 18 PCR products were sequenced. These 18 PCR products were selected as being representative of all anatomical sites of sampled patients and all hospital clinical wards during the study period. Twelve GREfm isolates were selected because they shared the same PFGE type A and subtypes (A1, A3, A5-A7, A10, A15, A16, A20, and A26) and were isolated at various times over the study period. The six GREfs isolates were chosen because of their different PFGE profiles (B-G) showing a difference of at least six bands from PFGE pattern A [33].

The restriction profiles of GREfm strains, presented in the dendrogram (Figure 1), show that the 12 selected pulsotype A strains belong to subtypes A1, A3, A5-A7, A10, A15, A16, A20 and A26. These subtypes correspond to 3 clones. The clone 2 included 7 strains belonging to 
TABLE 5: Characteristics of glycopeptide-resistant isolates.

\begin{tabular}{|c|c|c|c|c|c|c|c|}
\hline \multirow[t]{2}{*}{ Isolate type } & \multirow[t]{2}{*}{ Phenotype Van } & \multicolumn{4}{|c|}{ Susceptibility data (MIC- $\mu \mathrm{g} / \mathrm{mL}$ ) } & \multirow[t]{2}{*}{ PFGE type } & \multirow[t]{2}{*}{ Virulence factors } \\
\hline & & Penicillin & Ampicillin & Vancomycin & Teicoplanin & & \\
\hline & A & 3 & 1.5 & $>256$ & 32 & B & $\mathrm{Gel} \mathrm{E}^{+} \mathrm{Asal}^{+}$ \\
\hline \multirow[t]{6}{*}{ GRE faecalis $n=7$} & A & 3 & 1.5 & $>256$ & 32 & $\mathrm{C}$ & $\mathrm{Gel} \mathrm{E}^{+} \mathrm{Asa1}^{+}$ \\
\hline & A & 3 & 0.50 & $>256$ & 32 & $\mathrm{D}$ & $\mathrm{Gel} \mathrm{E}^{+}$ \\
\hline & A & 3 & 0.75 & $>256$ & 32 & $\mathrm{E}$ & $\mathrm{Gel} \mathrm{E}^{+}$ \\
\hline & A & 1.5 & 1.5 & $>256$ & $>256$ & $\mathrm{~F}$ & $\mathrm{Gel} \mathrm{E}^{+}$ \\
\hline & A & 2 & 1.5 & $>256$ & 32 & G & ${\mathrm{Asa} 1^{+}}^{+}$ \\
\hline & A & 4 & 1 & $>256$ & 32 & G & $\mathrm{Gel} \mathrm{E}^{+}$Asa $1^{+}$ \\
\hline GRE faecium & $39(29.8 \%) \mathrm{A}$ & 96 to $>256$ & 48 to $>256$ & $>256$ & 32 to $>256$ & A & $\mathrm{Hyl}^{+}$ \\
\hline$n=131$ & $92(70.2 \%) \mathrm{A}$ & 96 to $>256$ & 48 to $>256$ & $>256$ & 32 to $>256$ & A & $\mathrm{Hyl}^{+} \mathrm{Esp}^{+}$ \\
\hline
\end{tabular}

Asa1: Aggregation substance; gel E: Gelatinase, cylA: Cytolysin; esp: Enterococcal surface protein; hyl: Hyaluronidase.
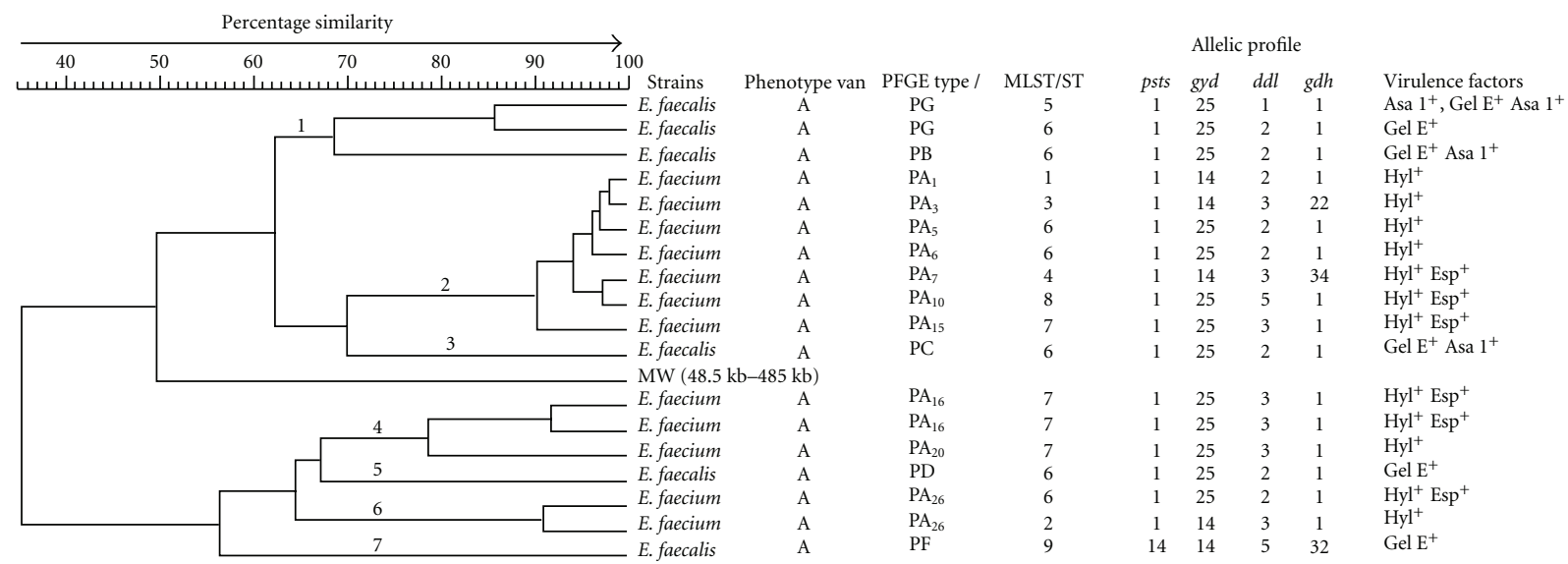

FIGURE 1: PFGE dendrogram with the corresponding MLST sequence types of the GRE isolates, digested with SmaI. Strains were clustered by the unweighted pair-group method with arithmetic mean (UPGMA). Each row represents a unique PFGE type with its unique PFGE pattern. The corresponding MLST sequence type (ST) is shown with the corresponding strains. Molecular weight: (MW). Bacteriophage $\lambda$-ladder is given in kbp (48.5 kb-485.0 kb) *PFGE type A (PA) isolates 3, 4, 6-8, 10-13-24, 27, 29-32, 34, 36, 37, 39-43, 45, 46, 49-53, 55-60, 63-101, 103-115, 117-138. (+): positive.

subtypes A1, A3, A5, A6, A7, A10, and A15. The percentage of similarity between each strain was between $90 \%$ and $98 \%$. The 3 strains of clone 4 belong to subtypes A16 and A20 and present the percentage of similarity between $78 \%$ and $91 \%$. Finally, the 2 strains of clone 6 belong to subtype A26 and have a percentage of similarity of $90 \%$. In total, the 12 subtypes A presenting a percentage of similarity between $78 \%$ and $98 \%$ show the propagation of one GREfm clone in hospitals of the Picardy region. The 6 GREfs pulsotypes analysis allowed to identify 4 clones. Clone 1 included 3 strains which present a percentage of similarity between $70 \%$ and $86 \%$, two of these strains belonging to pulsotype G have $86 \%$ of homology and the remaining one strain belonging to pulsotype B has $70 \%$ of homology. The strains of clones 3,5, and 7 belonging to pulsotypes $\mathrm{C}, \mathrm{D}$, and $\mathrm{F}$ have $72 \%, 67 \%$, and $57 \%$ of homology, respectively. These strains were isolated from patients hospitalized at nephrology, orthopedic surgery, thoracic surgery, and endocrinology wards and two patients from hepatology and gastroenterology wards. The dendrogram (Figure 1) may let us fear the emergence of other ERGfs clones as well as the dispersion of these bacteria following an epidemic mode, as seems to be the case of clone 1.

MLST analysis of the 18 isolates revealed 9 STs (Figure 1). The sequence types most frequently identified were ST6 (7 isolates) and ST7 (4 isolates) which shared the four housekeeping alleles, while ST1 (1 isolate), ST2 (1 isolate), ST3 (1 isolate), ST4 (1 isolate), ST5 (1 isolate), and ST8 (1 isolate) shared three of the four housekeeping alleles. The majority of these isolates belonged to the E. faecium species. They were clustered together with PFGE and were, therefore, considered to belong to the same pulsotype A. They were involved in a sustained outbreak in the hospitals of the Picardy region. Of six E. faecalis isolates, five were genotypically different and corresponded to 3 different STs (ST5, ST6, and ST9) (Figure 1). These STs differed from each other at one or four loci. 


\section{Discussion}

GRE are distributed worldwide, but their epidemiology appears to vary on a regional basis. Thus, polyclonal isolates were described [34], whereas some European Centres have reported nosocomial outbreak of GRE associated with very diverse epidemiological situations [3]. The data presented in this study show that most of the hospital-derived GREfm are part of a single clonal (pulsotype A). Among the acquired genes conferring resistance to glycopeptides, van $A$ is the only identified determinant. The use of multiplex PCR allowed simultaneous detection of enterococcal genes that encode for aggregation substance (asa 1), gelatinase (gel E), cytolysin (cyl A), enterococcal surface protein (esp), and hyaluronidase (hyl). In $131 \mathrm{GREfm}$ isolates, the asa 1 and $\mathrm{gel} E$ genes were not detected in this study which is in agreement with the results reported by other investigators $[13,35]$. In contrast, these genes were found in GREfs.

The combined presence of $h y l$ and esp genes was found in $70.2 \%$ of 131 GREfm isolates tested, which is in accordance with the findings of Vankerckhoven et al. [13] and Rice et al. [22]. In contrast, the only esp gene was not detected, as described elsewhere $[13,36]$. The $h y l$ gene was found in $29.8 \%$ of the 131 GREfm isolates, which is in contrast with the findings of Vankerckhoven et al. [13], who detected hyl gene in only $17 \%$ of the E. faecium isolates collected.

PFGE has been proposed as the method of choice from epidemiological typing of GREfm [37], although alternative technique, such as MLST analysis, has also been used successfully for characterization of GRE isolates [33]. The findings obtained by PFGE regarding the clonality of GREfm isolates were in accordance with MLST typing. A subpopulation of E. faecium adapted to the hospital setting, and consisting of strains responsible for epidemics, was characterized. The 12 representative GREfm isolates of pulsotype A analyzed by MLST belonged to ST1-ST4, ST6-ST8. These isolates are characterized by the presence of Hyl and Esp virulence factors as GREfm markers and high-level resistance to penicillin, ampicillin, vancomycin, and teicoplanin, in accordance with data of the literature [38, 39]. Top et al. [40] showed that their epidemic strains belonging to certain STs have been grouped in clonal complex 17 (CC17) of E. faecium. CC17 was defined upon MLST and is characterized by resistance to quinolones and ampicillin and the presence of the enterococcal surface protein (Esp) in the majority of isolates. The Hyl and Esp virulence factors have also been detected in vancomycinsusceptible strains [10]. The hypothesis proposed to explain the widespread distribution is the emergence of epidemic strains adapted to the hospital setting, which acquired vancomycin resistance determinants by horizontal gene transfer.

In this study, PFGE demonstrated the existence of a GREfm epidemic clone within the E. faecium population. The allelic profiles of this clone are relatively homogeneous, which suggests that they are genetically related. The GREfs isolates investigated by MLST were grouped into six different PFGE genogroups. This genetic diversity may not have emerged in the E. faecium epidemic population.
In conclusion, our data indicate that GREfm van A strains remain predominant in our region among GRE isolates, unlike that of the next North region of France, where an E. faecium vanB outbreak has been reported [6]. The recent increase in the number of GREfm in hospitals of the Picardy region might be due to the appearance and spread of a hospital-adapted, multidrug-resistant GREfm clone belonging to an internationally disseminated lineage. Horizontal gene transfer and clonal spread may both have contributed to the high rate of GREfm colonizations/infections. The divisions of colonized/infected patients into sectors, and an increased surveillance during the rehospitalizations of these patients, allow for the circumscription of the epidemic.

\section{Acknowledgment}

The authors would like to thank the hospitals of the Picardy region for their contribution to this study.

\section{References}

[1] R. C. Moellering Jr., "Emergence of Enterococcus as a significant pathogen," Clinical Infectious Diseases, vol. 14, no. 6, pp. 1173-1178, 1992.

[2] B. E. Murray, "Vancomycin-resistant enterococcal infections," New England Journal of Medicine, vol. 342, no. 10, pp. 710-721, 2000.

[3] G. Werner, T. M. Coque, A. M. Hammerum et al., "Emergence and spread of vancomycin resistance among Enterococci in Europe," Eurosurveillance, vol. 13, no. 47, pp. 1-11, 2008.

[4] A. H. C. Uttley, C. H. Collins, J. Naidoo, and R. C. George, "Vancomycin-resistant Enterococci," Lancet, vol. 1, no. 85758576, pp. 57-58, 1988.

[5] InVS (Institut de Veille Sanitaire), "Contrôle des entérocoques résistants aux glycopeptides (ERG): état des lieux en France," Bulletin Epidémiologique Hebdomadaire, no. 41-42, pp. 385407, 2008.

[6] N. Bourdon, M. Fines, and R. Leclercq, "Caractéristiques des souches d'entérocoques résistants aux glycopeptides isolées en France, 2006-2008," Bulletin Epidémiologique Hebdomadaire, no. 41-42, pp. 391-394, 2008.

[7] C. H. Heath, T. K. Blackmore, and D. L. Gordon, "Emerging resistance in Enterococcus spp.," Medical Journal of Australia, vol. 164, no. 2, pp. 116-120, 1996.

[8] S. Al-Obeid, L. Gutmann, D. M. Shlaes, R. Williamson, and E. Collatz, "Comparison of vancomycin-inducible proteins from four strains of Enterococci," FEMS Microbiology Letters, vol. 58, no. 1, pp. 101-105, 1990.

[9] A. H. C. Uttley, R. C. George, J. Naidoo et al., "High-level vancomycin-resistant Enterococci causing hospital infections," Epidemiology and Infection, vol. 103, no. 1, pp. 173-181, 1989.

[10] R. Quintiliani Jr., S. Evers, and P. Courvalin, "The vanB gene confers various levels of self-transferable resistance to vancomycin in Enterococci," Journal of Infectious Diseases, vol. 167, no. 5, pp. 1220-1223, 1993.

[11] P. Courvalin, "Vancomycin resistance in gram-positive cocci," Clinical Infectious Diseases, vol. 42, no. 1, pp. S25-S34, 2006.

[12] S. J. McKessar, A. M. Berry, J. M. Bell, J. D. Turnidge, and J. C. Paton, "Genetic characterization of van $G$, a novel vancomycin resistance locus of Enterococcus faecalis," Antimicrobial Agents and Chemotherapy, vol. 44, no. 11, pp. 3224-3228, 2000. 
[13] V. Vankerckhoven, T. van Autgaerden, C. Vael et al., "Development of a multiplex PCR for the detection of asaI, gelE, cylA, esp, and hyl genes in Enterococci and survey for virulence determinants among European hospital isolates of Enterococcus faecium," Journal of Clinical Microbiology, vol. 42, no. 10, pp. 4473-4479, 2004.

[14] D. Galli, F. Lottspeich, and R. Wirth, "Sequence analysis of Enterococcus faecalis aggregation substance encoded by the sex pheromone plasmid pAD1," Molecular Microbiology, vol. 4, no. 6, pp. 895-904, 1990.

[15] B. Kreft, R. Marre, U. Schramm, and R. Wirth, "Aggregation substance of Enterococcus faecalis mediates adhesion to cultured renal tubular cells," Infection and Immunity, vol. 60, no. 1, pp. 25-30, 1992.

[16] C. A. Guzman, C. Pruzzo, G. LiPira, and L. Calegari, "Role of adherence in pathogenesis of Enterococcus faecalis urinary tract infection and endocarditis," Infection and Immunity, vol. 57, no. 6, pp. 1834-1838, 1989.

[17] Y. A. Su, M. C. Sulavik, P. He et al., "Nucleotide sequence of the gelatinase gene (gelE) from Enterococcus faecalis subsp. liquefaciens," Infection and Immunity, vol. 59, no. 1, pp. 415420, 1991.

[18] J. W. Chow, L. A. Thal, M. B. Perri et al., "Plasmidassociated hemolysin and aggregation substance production contribute to virulence in experimental enterococcal endocarditis," Antimicrobial Agents and Chemotherapy, vol. 37, no. 11, pp. 2474-2477, 1993.

[19] B. D. Jett, M. M. Huycke, and M. S. Gilmore, "Virulence of Enterococci," Clinical Microbiology Reviews, vol. 7, no. 4, pp. 462-478, 1994.

[20] V. Shankar, A. S. Baghdayan, M. M. Huycke, G. Lindahl, and M. S. Gilmore, "Infection-derived Enterococcus faecalis strains are enriched in esp, a gene encoding a novel surface protein," Infection and Immunity, vol. 67, no. 1, pp. 193-200, 1999.

[21] N. Shankar, C. V. Lockatell, A. S. Baghdayan, C. Drachenberg, M. S. Gilmore, and D. E. Johnson, "Role of Enterococcus faecalis surface protein esp in the pathogenesis of ascending urinary tract infection," Infection and Immunity, vol. 69, no. 7, pp. 4366-4372, 2001.

[22] L. B. Rice, L. Carias, S. Rudin et al., "A potential virulence gene, hylEfm, predominates in Enterococcus faecium of clinical origin," Journal of Infectious Diseases, vol. 187, no. 3, pp. 508$512,2003$.

[23] A. M. Berry and J. C. Paton, "Additive attenuation of virulence of Streptococcus pneumoniae by mutation of the genes encoding pneumolysin and other putative pneumococcal virulence proteins," Infection and Immunity, vol. 68, no. 1, pp. 133-140, 2000.

[24] Comité de l'Antibiogramme de la Société Française de Microbiologie (CA-SFM)—Recommandations, 2009.

[25] S. Dutka-Malen, S. Evers, and P. Courvalin, "Detection of glycopeptide resistance genotypes and identification to the species level of clinically relevant Enterococci by PCR," Journal of Clinical Microbiology, vol. 33, no. 1, pp. 24-27, 1995.

[26] H. Leavis, J. Top, N. Shankar et al., "A novel putative enterococcal pathogenicity island linked to the esp virulence gene of Enterococcus faecium and associated with epidemicity," Journal of Bacteriology, vol. 186, no. 3, pp. 672-682, 2004.

[27] J.-P. Lavigne, H. Marchandin, E. Czarnecki, C. Kaye, and A. Sotto, "Bactériémies à Enterococcus spp.: Étude prospective au CHU de Nîmes," Pathologie Biologie, vol. 53, no. 8-9, pp. 539$545,2005$.
[28] F. C. Tenover, R. D. Arbeit, R. V. Goering et al., "Interpreting chromosomal DNA restriction patterns produced by pulsedfield gel electrophoresis: criteria for bacterial strain typing," Journal of Clinical Microbiology, vol. 33, no. 9, pp. 2233-2239, 1995.

[29] W. L. Homan, D. Tribe, S. Poznanski et al., "Multilocus sequence typing scheme for Enterococcus faecium," Journal of Clinical Microbiology, vol. 40, no. 6, pp. 1963-1971, 2002.

[30] CTNIN (Comité Technique National des Infections Nosocomiales), 100 Recommandations pour la Surveillance et la Prévention des Infections Nosocomiales, Ministère de l'Emploi et de la Solidarité, Secrétaire d'Etat à la Santé et l'Action Sociale, Paris, France, 2nd edition, 1999.

[31] J. S. Garner, W. R. Jarvis, T. G. Emori, T. C. Horan, and J. M. Hughes, "CDC defionitions for nosocomial infections, 1988," American Journal of Infection Control, vol. 16, no. 3, pp. 128140, 1988.

[32] L. Stampone, M. Del Grosso, D. Boccia, and A. Pantosti, "Clonal spread of a vancomycin-resistant Enterococcus faecium strain among bloodstream-infecting isolates in Italy," Journal of Clinical Microbiology, vol. 43, no. 4, pp. 1575-1580, 2005.

[33] L. L. Nemoy, M. Kotetishvili, J. Tigno et al., "Multilocus sequence typing versus pulsed-field gel electrophoresis for characterization of extended-spectrum $\beta$-lactamaseproducing Escherichia coli isolates," Journal of Clinical Microbiology, vol. 43, no. 4, pp. 1776-1781, 2005.

[34] M. Kolar, R. Pantucek, I. Vagnerova et al., "Genotypic characterisation of vancomycin-resistant Enterococcus faecium isolates from haemato-oncological patients at Olomouc University Hospital, Czech Republic," Clinical Microbiology and Infection, vol. 12, no. 4, pp. 353-360, 2006.

[35] I. Duprè, S. Zanetti, A. M. Schito, G. Fadda, and L. A. Sechi, "Incidence of virulence determinants in clinical Enterococcus faecium and Enterococcus faecalis isolates collected in Sardinia (Italy)," Journal of Medical Microbiology, vol. 52, no. 6, pp. 491-498, 2003.

[36] T. J. Eaton and M. J. Gasson, "A variant enterococcal surface protein $\operatorname{Esp}_{f m}$ in Enterococcus faecium; distribution among food, commensal, medical, and environmental isolates," FEMS Microbiology Letters, vol. 216, no. 2, pp. 269-275, 2002.

[37] T. Kuriyama, D. W. Williams, M. Patel et al., "Molecular characterization of clinical and environmental isolates of vancomycin-resistant Enterococcus faecium and Enterococcus faecalis from a teaching hospital in Wales," Journal of Medical Microbiology, vol. 52, no. 9, pp. 821-827, 2003.

[38] R. J. L. Willems, J. Top, M. van Santen et al., "Global spread of vancomycin-resistant Enterococcus faecium from distinct nosocomial genetic complex," Emerging Infectious Diseases, vol. 11, no. 6, pp. 821-828, 2005.

[39] R. J. L. Willems, W. Homan, J. Top et al., "Variant esp gene as a marker of a distinct genetic lineage of vancomycin-resistant Enterococcus faecium spreading in hospitals," Lancet, vol. 357, no. 9259, pp. 853-855, 2001.

[40] J. Top, R. Willems, S. van der Velden, M. Asbroek, and M. Bonten, "Emergence of clonal complex 17 Enterococcus faecium in the Netherlands," Journal of Clinical Microbiology, vol. 46, no. 1, pp. 214-219, 2008. 

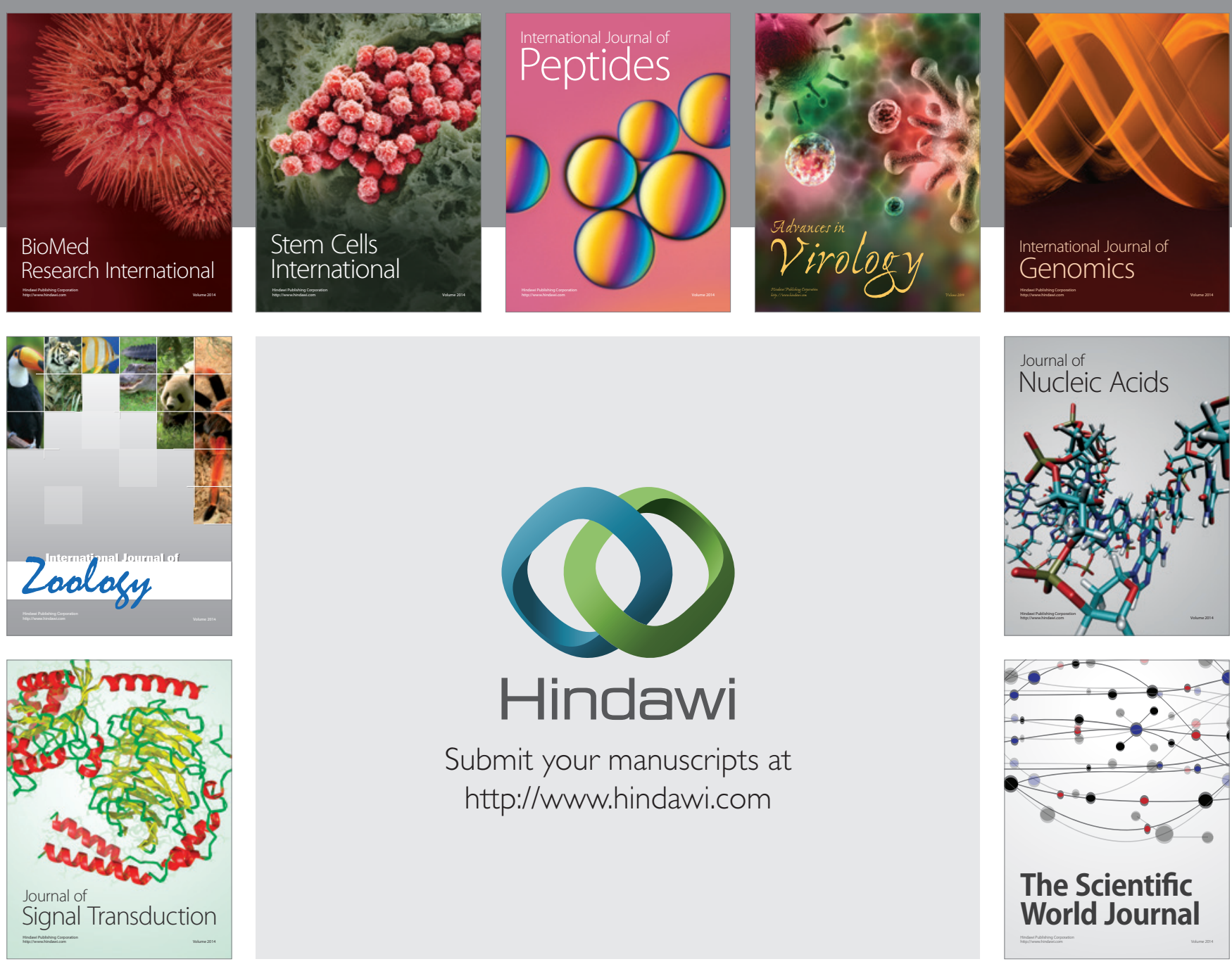

Submit your manuscripts at

http://www.hindawi.com
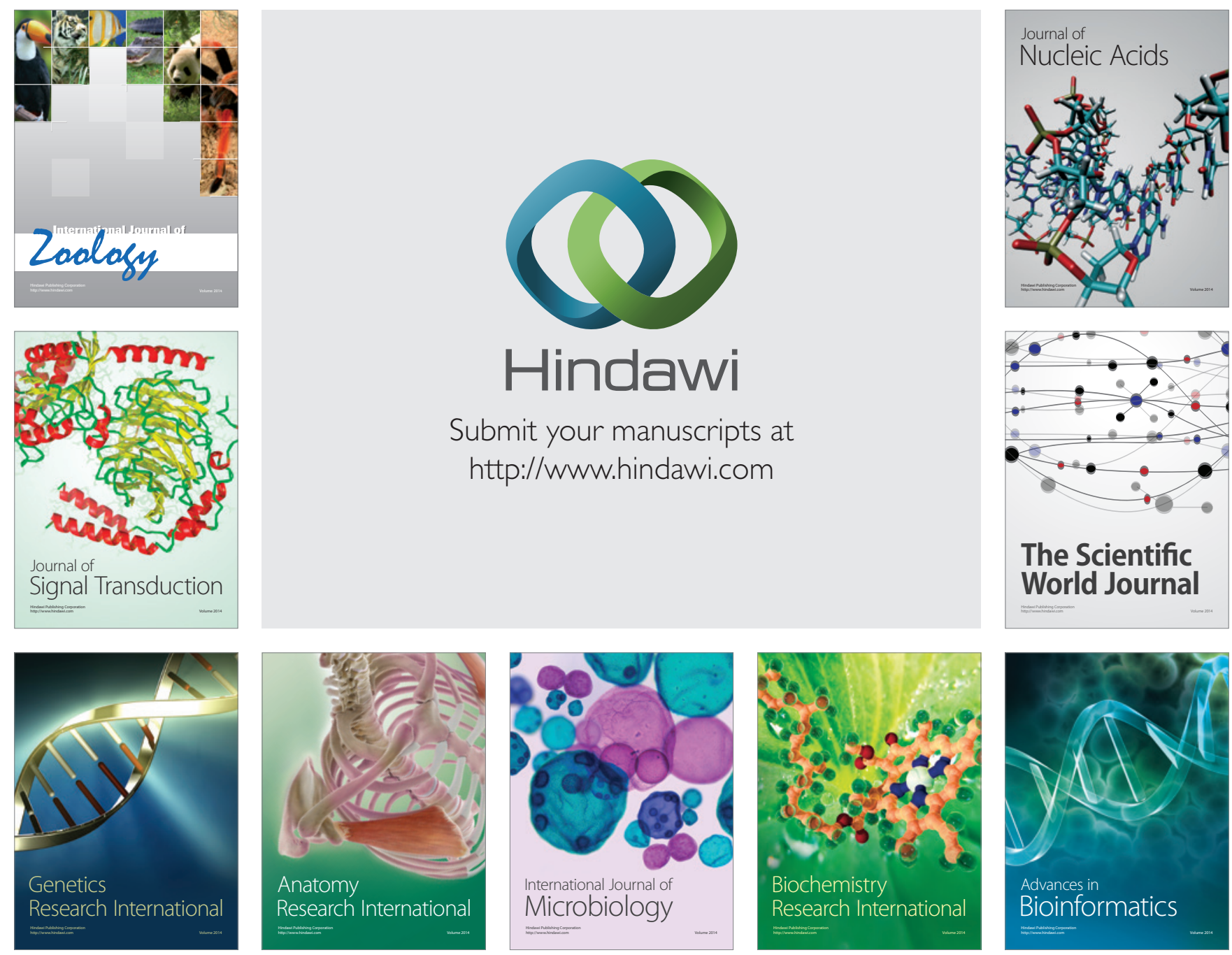

The Scientific World Journal
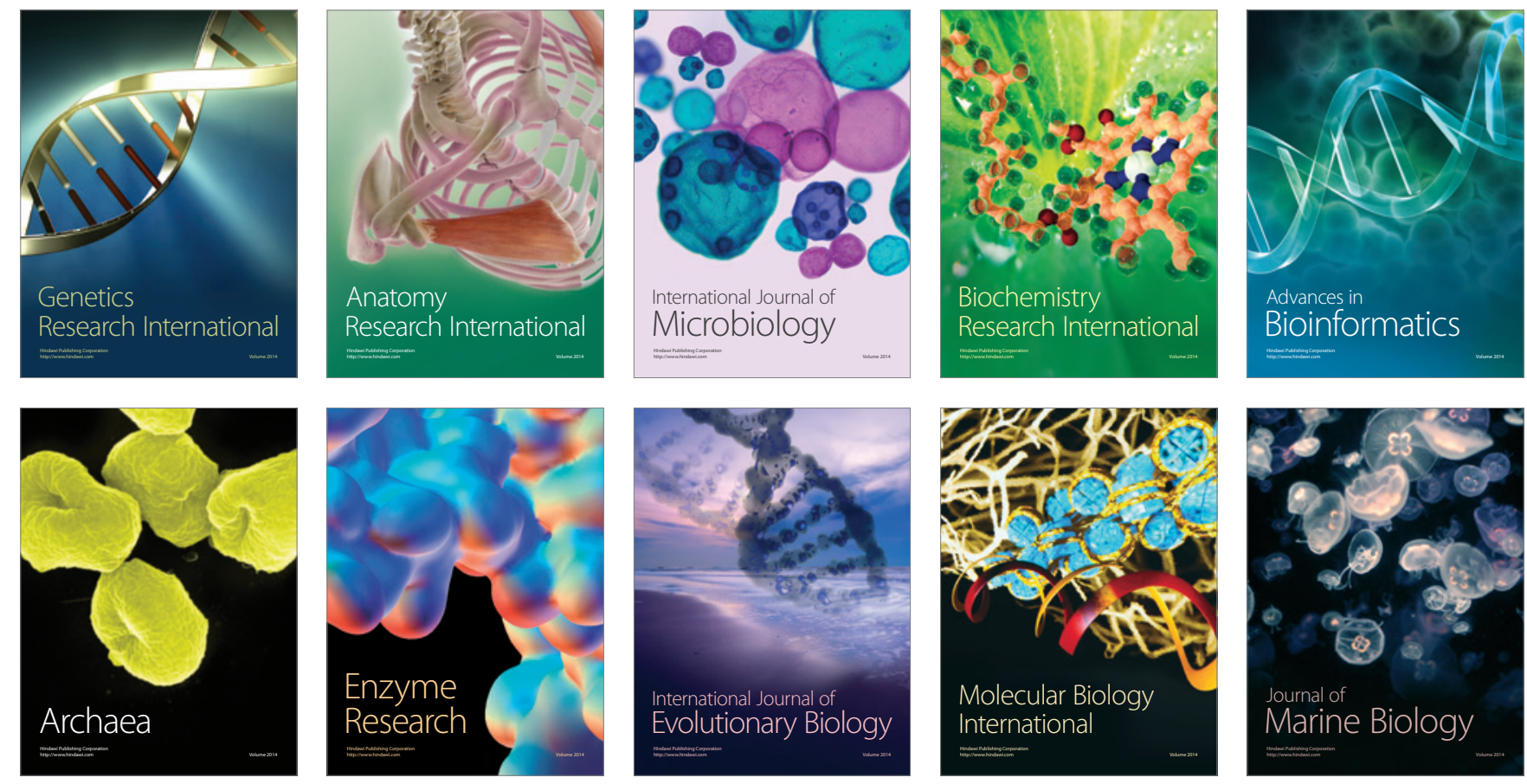